\title{
New dynamical behavior of two waves for $(2+1)$-dimensional Broer-Kaup equation
}

\author{
Ying Jiang ${ }^{\mathrm{a}, *}$, Da-Quan Xian ${ }^{\mathrm{a}}$, Zheng-De Dai ${ }^{\mathrm{b}}$ \\ a School of Science, Southwest University of Science and Technology, Mianyang, 621010, P. R. China. \\ ${ }^{b}$ School of Mathematics and Statistics, Yunnan University, Kunming 650091, P. R. China. \\ Communicated by $\mathrm{X}$. J. Yang
}

\begin{abstract}
New exact solutions including periodic breather wave, kink breather wave and doubly breather wave solutions are obtained for $(2+1) \mathrm{D}$ BK equation by using Painleve analysis, variable separation approach, the homoclinic test method and generalized CK method via the linearization of equation, variable separation and equivalent transformation, respectively. The dynamical behavior and interaction between different waves are investigated. These results enrich the dynamic features of higher dimensional nonlinear system. (C)2017 All rights reserved.
\end{abstract}

Keywords: Broer-Kaup equation, coincidence point, equivalent transformation, variable separation, generalized CK method, dynamical behavior.

2010 MSC: 35Q51, 35Q53, 35Q99.

\section{Introduction}

Many nonlinear phenomena in nature and human society are usually characterized by nonlinear evolution equations. Searching for an analytical exact solution to a nonlinear system becomes one of the central themes of perpetual interest in nonlinear science. Various methods for obtaining exact solutions of a nonlinear system have been proposed, such as the Hirota bilinear method, the homogeneous balance method, inverse scattering method, Backlund transformation, variable separation approach, Painleve analysis method, Darboux transformation, symmetry reduction method, homoclinic test method, the extended homoclinic test method [2] and so on. The $(2+1)$-dimensional Broer-Kaup Equation $((2+1) \mathrm{D} B K$ for short) comes from the constraints of the KP equation and it is of importance in mathematical physics field. Many researchers pay more and more attention to search for analytical exact solution to (2+1)D BK Equation because of its rich physical connotation [1, 3-14, 16-24]. By means of the homogeneous balance method [8, 16-19, 21, 24] solitary wave solutions, exact multi-soliton solutions and soliton-like solutions of the BK equation were obtained. Meanwhile, doubly periodic wave solutions, folded solitary wave solutions, non-Lie symmetry groups and new exact solutions were derived [6] by using variable separation

\footnotetext{
${ }^{*}$ Corresponding author Dai)

Email addresses: xsjy2000@qq.com (Ying Jiang), xiandaquan@swust.edu.cn (Da-Quan Xian), zddai@ynu.edu.cn (Zheng-De
} 
approach $[1,3,11,12,15,22]$, Painleve analysis method [5, 20] and generalized Riccati mapping method $[10,23]$. In this work, we consider the following $(2+1)$ D BK Equation:

$$
\left\{\begin{array}{l}
u_{t y}-u_{x x y}+2\left(u u_{x}\right)_{y}+2 v_{x x}=0 \\
v_{t}+v_{x x}+2(u v)_{x}=0
\end{array}\right.
$$

We will study on the new exact solutions including periodic breather wave, kink breather wave and doubly breather wave solutions by Painleve analysis, variable separation approach, homoclinic test method, generalized CK method and equivalent transformation via the linearization of equation, respectively. The dynamical behavior and interaction between different waves are investigated.

\section{The linearization of Equation (1.1)}

Substituting $v=u_{y}$ into (1.1), we get

$$
u_{t y}+u_{x x y}+\left(u^{2}\right)_{x y}=0
$$

integrating with respect to $y$ and set the integration constant to zero, we can obtain

$$
u_{t}+u_{x x}+\left(u^{2}\right)_{x}=0 .
$$

Equation (2.1) is a Burgers type equation. Based on the Painleve analysis, we take the following transformation

$$
u=(\ln \varphi)_{x}
$$

Substituting (2.2) into (2.1) and integrating with respect to $x$, we get the following second-order LPDE.

$$
\varphi_{\mathrm{t}}+\varphi_{x \mathrm{x}}=0 \text {, }
$$

where $\varphi$ is function of variable $(x, y, t)$ to be determined. Based on the solutions of (2.3), we can obtain the exact solutions for the BK system.

\section{Exact solutions of the Equation (1.1)}

From the transformation (2.2), we assume the variable separation solutions in this form

$$
\varphi=f(x, t)+g(y),
$$

where $f(x, t)$ is function of $(x, t)$ to be determined later and $g(y)$ is arbitrary function of $y$, respectively. Substituting (3.1) into (2.3), we have

$$
f_{t}(x, t)+f_{x x}(x, t)=0 .
$$

This is a famous heat conduction equation. Next, we will derive two types of variable separation solutions of (3.2).

3.1. Variable separation solutions with sum-form

Now, we suppose the solution of (3.2) as

$$
f(x, t)=p(x)+q(t) .
$$

Substituting (3.3) into (2.3), we get the following results:

$$
q(t)=c_{0} t, \quad p(x)=\frac{-c_{0} x^{2}}{2}+c_{1} x+c_{2}, \quad \varphi_{1}=\frac{c_{0} x^{2}}{2}-c_{1} x-c_{0} t+c_{2}+g(y),
$$

where $c_{0}, c_{1}, c_{2}$ are arbitrary constants.

\subsection{Variable separation solutions with product-form}

We suppose the solution of (3.2) as $f(x, t)=p(x) q(t)$. Substituting it into (3.2), we get the following results:

$$
p(x)=c_{1} e^{c_{0} x}+c_{2} e^{-c_{0} x},
$$


or

$$
\begin{gathered}
p(x)=\sinh \left(c_{0} x\right), \text { or } p(x)=\cosh \left(c_{0} x\right), q(t)=e^{-c_{0}^{2} t}, \\
\varphi_{2}=e^{-c_{0}^{2} t} \cosh \left(c_{0} x\right)+g(y) .
\end{gathered}
$$

Based on linear superposition principle of solutions of linear equations, the following superposition solutions are available

$$
\varphi_{3}=c_{3}\left(\frac{c_{0} x^{2}}{2}-c_{1} x-c_{0} t+c_{2}\right)+c_{4}\left(e^{-c_{0}^{2} t} \cosh \left(c_{0} x\right)\right)+g(y),
$$

where $c_{i} \in R(i=0, \cdots, 4)$. Substituting (3.4), (3.5), (3.6) into (2.2) and notice $v=u_{y}$, we obtain the exact solutions of (1.1) as follows:

$$
\begin{gathered}
u_{1}=\frac{c_{0} x-c_{1}}{\frac{c_{0} x^{2}}{2}-c_{1} x-c_{0} t+c_{2}+g(y)}, \quad v_{1}=\frac{-\left(c_{0} x-c_{1}\right) g^{\prime}(y)}{\left[\frac{c_{0} x^{2}}{2}-c_{1} x-c_{0} t+c_{2}+g(y)\right]^{2}}, \\
u_{2}=\frac{c_{0} e^{-c_{0}^{2} t} \sinh \left(c_{0} x\right)}{e^{-c_{0}^{2} t} \cosh \left(c_{0} x\right)+g(y)}, \quad v_{2}=\frac{-c_{0} e^{-c_{0}^{2} t} \sinh \left(c_{0} x\right) g^{\prime}(y)}{\left[e^{-c_{0}^{2} t} \cosh \left(c_{0} x\right)+g(y)\right]^{2}}, \\
\left\{\begin{array}{l}
u_{3}=\frac{c_{0} x-c_{1}-c_{0} e^{-c_{0}^{2} t} \sinh \left(c_{0} x\right)}{\frac{c_{0} x^{2}}{2}-c_{1} x-c_{0} t+c_{2}+e^{-c_{0}^{2} t} \cosh \left(c_{0} x\right)+g(y)}, \\
v_{3}=\frac{-\left[c_{0} x-c_{1}-c_{0} e^{-c_{0}^{2} t} \sinh \left(c_{0} x\right)\right] g^{\prime}(y)}{\left[\frac{c_{0} x^{2}}{2}-c_{1} x-c_{0} t+c_{2}+e^{-c_{0}^{2} t} \cosh \left(c_{0} x\right)+g(y)\right]^{2}} .
\end{array}\right.
\end{gathered}
$$

3.3. Interaction between periodic breather wave and single solitary wave

Based on the homoclinic test method, we suppose

$$
\varphi=e^{k_{1} x+s_{1} y+c_{1} t+r_{1}}+a \cos \left(k_{2} x+s_{2} y+c_{2} t+r_{2}\right)+b \cosh \left(k_{3} x+s_{3} y+c_{3} t+r_{3}\right),
$$

where $a, b, k_{i}, s_{i}, c_{i}, r_{i}(i=1,2,3)$ are constants to be determined later. Substituting (3.7) into (2.3), we obtain the set of algebraic equations for $a, b, k_{i}, s_{i}, c_{i}, r_{i}(i=1,2,3)$

$$
k_{2}^{3}+k_{1}^{2} k_{2}+c_{1} k_{2}+c_{2} k_{1}=0, \cdots, c_{3} k_{2}+c_{2} k_{3}=0, \quad c_{3} k_{3}-c_{2} k_{2}=0 .
$$

The solution of (3.8) is $c_{1}=-k_{1}^{2}+k_{3}^{2}$, when $a=c_{3}=0$. Thus we have

$$
\varphi_{4}=e^{k_{1} x+s_{1} y-\left(k_{1}^{2}-k_{3}^{2}\right) t+r_{1}}+b \cosh \left(k_{3} x+s_{3} y+r_{3}\right) .
$$

Substituting (3.9) into (2.2) and $v=u_{y}$ in turn, we can derive the following exact solutions of (1.1):

$$
\left\{\begin{array}{l}
u_{4}=\frac{k_{1} e^{k_{1} x+s_{1} y-\left(k_{1}^{2}-k_{3}^{2}\right) t+r_{1}}+b k_{3} \sinh \left(k_{3} x+s_{3} y+r_{3}\right)}{e^{k_{1} x+s_{1} y-\left(k_{1}^{2}-k_{3}^{2}\right) t+r_{1}}+b \cosh \left(k_{3} x+s_{3} y+r_{3}\right)}, \\
v_{4}=\frac{s_{1} e^{k_{1} x+s_{1} y-\left(k_{1}^{2}-k_{3}^{2}\right) t+r_{1}}+b s_{3} \sinh \left(k_{3} x+s_{3} y+r_{3}\right)}{\left[e^{k_{1} x+s_{1} y-\left(k_{1}^{2}-k_{3}^{2}\right) t+r_{1}}+b \cosh \left(k_{3} x+s_{3} y+r_{3}\right)\right]^{2}} .
\end{array}\right.
$$

\subsection{Equivalence transformation of Equation (2.3)}

Based on generalized CK method, we suppose $\varphi$ as

$$
\varphi=\alpha+\beta f(\xi, \eta)
$$

where $\alpha=\alpha(x, y, t), \beta=\beta(x, y, t), \xi=\xi(x, y, t), \eta=\eta(x, y, t)$ are functions of $(x, y, t)$ to be determined later and $f(\xi, \eta)$ is a solution of linear equations

$$
f_{\eta}+f_{\xi \xi}=0 .
$$

Substituting (3.10) and (3.11) into (2.3), we obtain

$$
\alpha=g(y), \quad \beta=1, \quad \xi=h(y) x, \quad \eta=h^{2}(y) t,
$$

where $h(y), g(y)$ are arbitrary functions of $y$. Substituting (3.12) into (3.10), we obtain equivalence transformation of (2.3)

$$
\varphi=f\left(h(y) x, h^{2}(y) t\right)+g(y) .
$$


Base on transformation (3.10) of (2.3), we obtain invariant form solutions,

$$
\begin{aligned}
& \varphi_{5}=\frac{c_{0} h^{2}(y) x^{2}}{2}-c_{1} h(y) x-c_{0} h^{2}(y) t+c_{2}+g(y), \\
& \varphi_{6}=h(y) \cosh \left(c_{0} h(y) x\right) e^{-c_{0} h^{2}(y) t}+g(y), \\
& \varphi_{7}=c_{3}\left[\frac{c_{0} h^{2}(y) x^{2}}{2}-c_{1} h(y) x-c_{0} h^{2}(y) t+c_{2}\right]+c_{4} h(y) e^{k^{2} h^{2}(y) t} \cosh \left(c_{0} h(y) x\right)+g(y) .
\end{aligned}
$$

We can derive the following exact solutions of (1.1) from (3.13), (3.14), (3.15):

$$
\begin{aligned}
& u_{5}=\frac{c_{0} h^{2}(y) x-c_{1} h(y)}{\frac{c_{0} h^{2}(y) x^{2}}{2}-c_{1} h(y) x-c_{0} h^{2}(y) t+c_{2}+g(y)}, \quad v_{5}=\left[\frac{c_{0} h^{2}(y) x-c_{1} h(y)}{\frac{c_{0} h^{2}(y) x^{2}}{2}-c_{1} h(y) x-c_{0} h^{2}(y) t+c_{2}+g(y)}\right]_{y}, \\
& u_{6}=\frac{c_{0} h^{2}(y) x-c_{1} h(y)}{\frac{c_{0} h^{2}(y) x^{2}}{2}-c_{1} h(y) x-c_{0} h^{2}(y) t+c_{2}+g(y)}, \quad v_{6}=\left[\frac{c_{0} h^{2}(y) x-c_{1} h(y)}{\frac{c_{0} h^{2}(y) x^{2}}{2}-c_{1} h(y) x-c_{0} h^{2}(y) t+c_{2}+g(y)}\right]_{y} \\
& \left\{\begin{array}{l}
u_{7}=\frac{c_{3}\left[c_{0} h^{2}(y) x-c_{1} h(y)\right]-c_{4} k h^{2}(y) e^{k^{2} h^{2}(y) t} \sin (k h(y) x}{c_{3}\left[c_{0} h^{2}(y)\left(\frac{x^{2}}{2}-t\right)-c_{1} h(y) x+c_{2}\right]+c_{4} h(y) e^{k^{2} h^{2}(y) t} \cos (k h(y) x+g(y)}, \\
v_{7}=\left[\frac{c_{3}\left[c_{0} h^{2}(y) x-c_{1} h(y)\right]-c_{4} k h^{2}(y) e^{k^{2} h^{2}(y) t} \sin (k h(y) x}{c_{3}\left[c_{0} h^{2}(y)\left(\frac{x^{2}}{2}-t\right)-c_{1} h(y) x+c_{2}\right]+c_{4} h(y) e^{k^{2} h^{2}(y) t} \cos (k h(y) x+g(y)}\right]_{y} .
\end{array}\right.
\end{aligned}
$$

\section{Dynamic behavior analysis of solution of Equation (1.1)}

In this section, we mainly discuss the dynamical behavior and localized structure of solution $u_{7}$ and $v_{7}$ for (1.1). $u_{7}$ and $v_{7}$ describe the behavior of inelastic collision between line soliton $\mathrm{g}(\mathrm{y})$ and different kink-type breather solitons. At the same time, they show different structures of solitons in different regions.

4.1 Interaction between breather wave and kink wave.

For example, if we choose $g(y)=y^{6}+1, h(y)=\tanh (y)+1, k=0.03, c_{i}=1(i=0, \cdots, 4)$, we can obtain the localized structure of $u_{7}$ and $v_{7}$ at time $t=-0.8, t=0, t=0.8$, respectively. They are expressed by Figure 1 in turn.
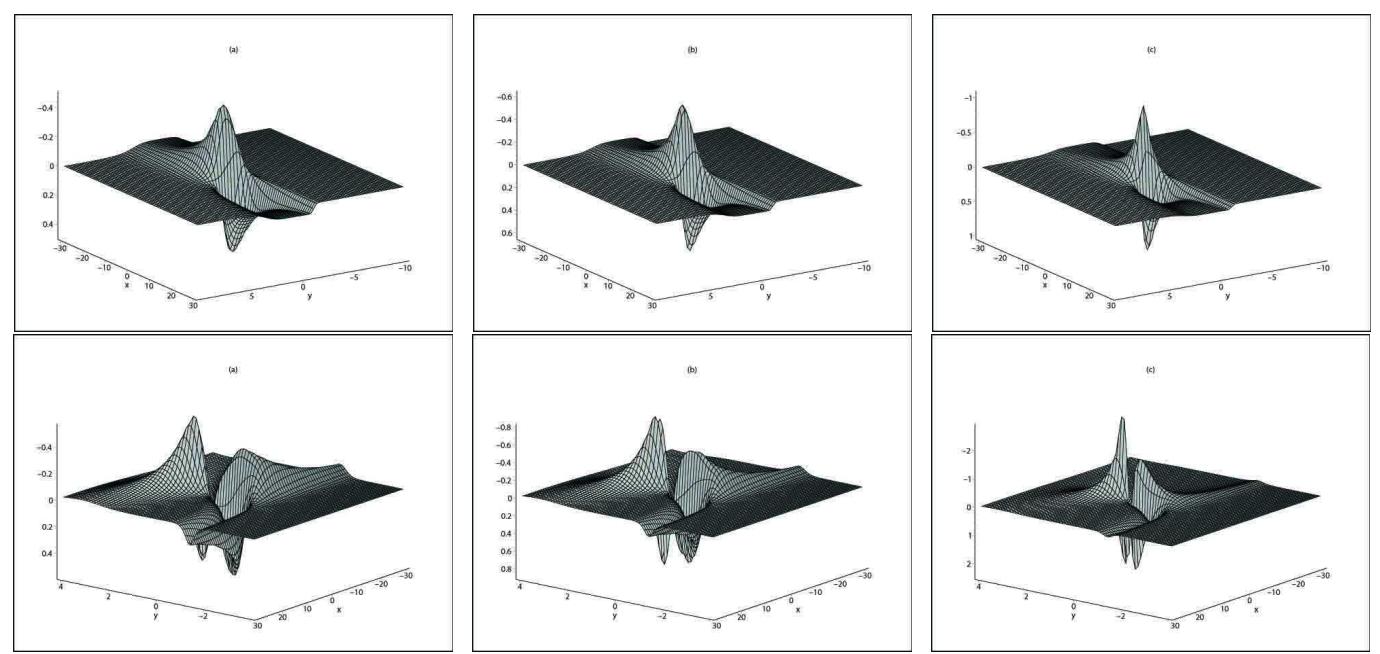

Figure 1: Row 1 Shows that $u_{7}$ is kink-type breather wave and it describes the interaction between kink wave and breather wave with different directions. Row 2 Shows that $v_{7}$ is doubly breather-type wave and it describes the interaction between kink wave and doubly breather-type wave with different directions. 


\subsection{Interaction between line solition and dromion solition.}

If we choose $g(y)=y^{4}+1, h(y)=y \sin \left(\ln \left(y^{2}\right)\right), k=0.11, c_{i}=1(i=0, \cdots, 4)$, we can obtain the localized structure of $u_{7}$ and $v_{7}$ at time $t=-3.6, t=0, t=3.6$, respectively. They are expressed by Figure 2 in turn.
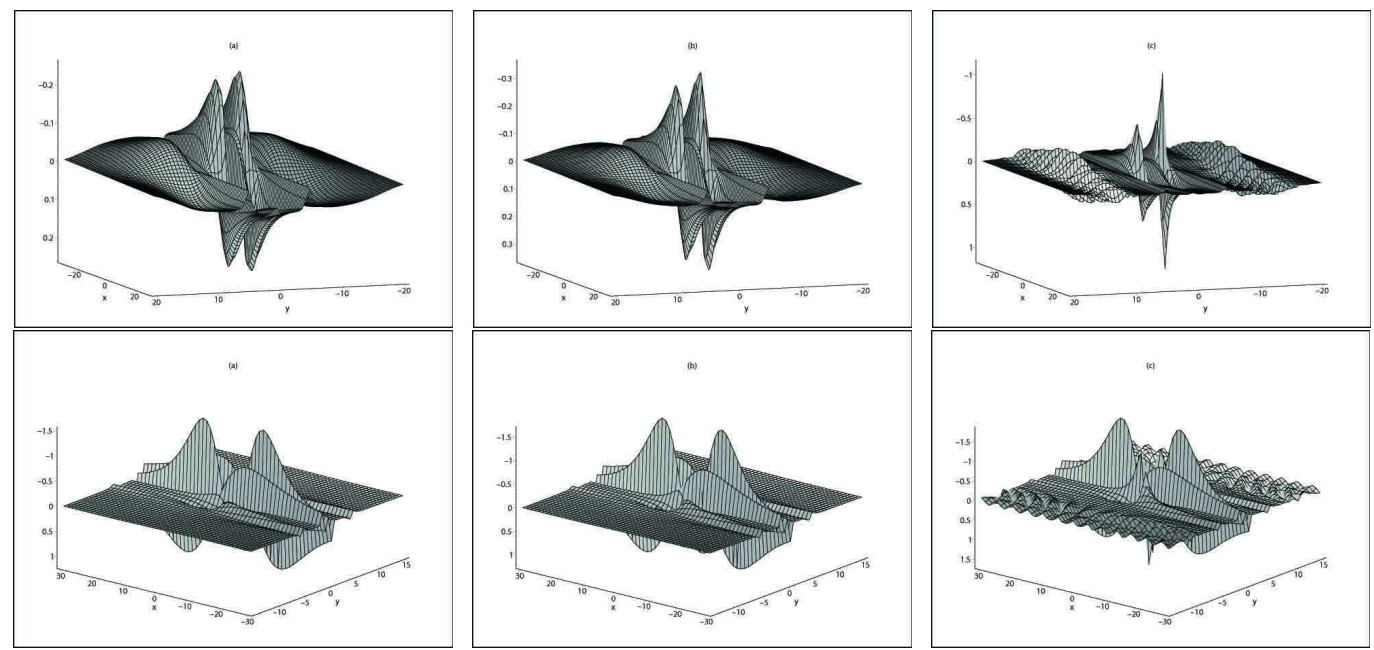

Figure 2: Row 1 and Row 2 show interaction between line solition and dromion solition, which is inelastic collision, and exhibit the evolution of the two solitons from the chaotic line solition to the chaotic structure.

\subsection{Interaction between line solition and compaction.}

If we choose $g(y)=\operatorname{sech}(y)+1, h(y)=\sin (y), k=0.3, c_{i}=1(i=0, \cdots, 4)$, we can obtain the localized structure of $u_{7}$ and $v_{7}$ at time $t=-0.5, t=0, t=0.5$, respectively. They are expressed by Figure 3 in turn.
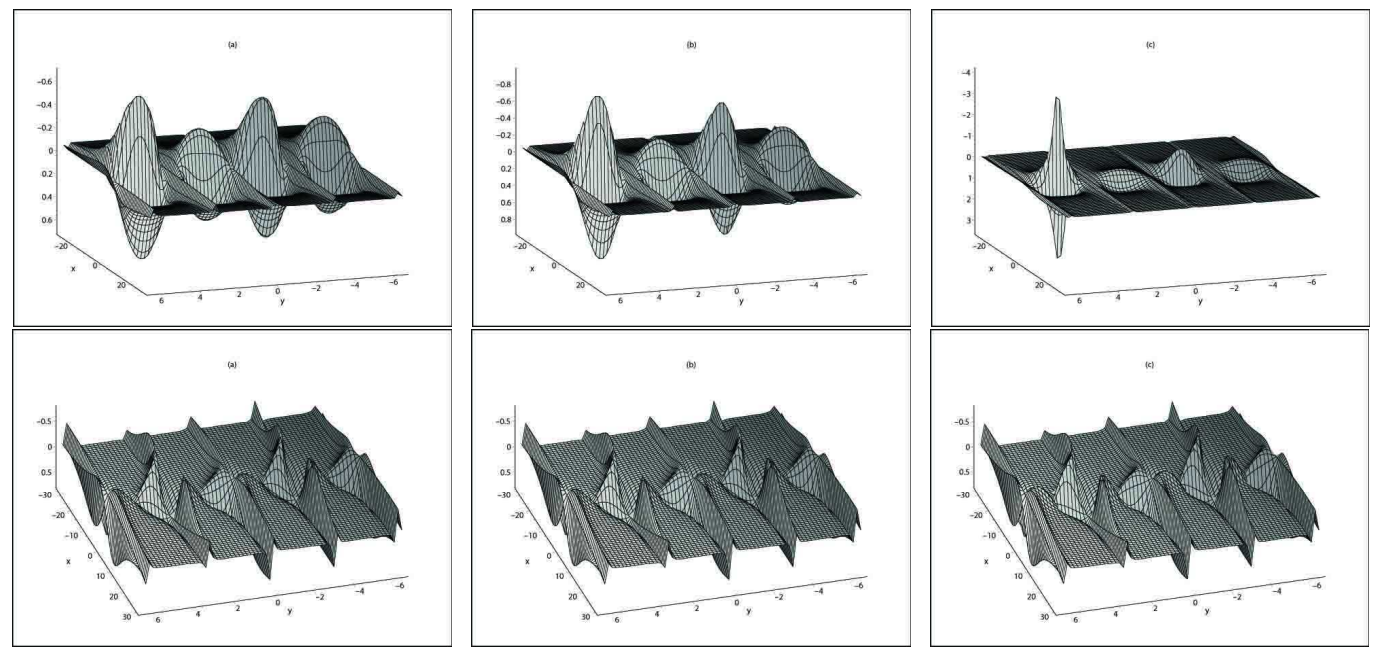

Figure 3: Row 1 and Row 2 show interaction between line solition and compaction, which are inelastic collision, and exhibit degradation of chaotic line soliton, but they have not chaotic structure. 


\section{Conclusions}

In this paper, we study the linearization of $(2+1) \mathrm{D}$ BK Equation and obtain some new exact solutions including periodic breather-type wave, kink breather wave and doubly breather wave using Painleve analysis, variable separation approach, the homoclinic test method and generalized CK method. Furthermore, we discuss its dynamical behavior and localized structure of interaction between breather wave and kink wave, line solition and dromion solution, line solition and compaction, etc. These results enrich the dynamic significance of the higher dimensional nonlinear system.

\section{Acknowledgment}

This work was supported by Chinese Natural Science Foundation Grant No. 11204250 , No. 11202175 and No. 11361048.

\section{References}

[1] C.-Q. Dai, X. Cen, S.-S. Wu, Exotic localized structures based on a variable separation solution of the (2+1)-dimensional higher-order Broer-Kaup system, Nonlinear Anal., 10 (2009), 259-261. 1

[2] Z.-D. Dai, J. Liu, D.-L. Li, Applications of HTA and EHTA to YTSF equation, Appl. Math. Comput., 207 (2009), 360-364. 1

[3] B.-J. Hong, D.-C. Lu, New soliton-like solutions to the $(2+1)$-dimensional Broer-Kaup equation with variable coefficients, J. At. Mol. Phys., 25 (2008), 130-134. 1

[4] W.-H. Huang, Y.-L. Liu, Z.-M. Lu, Doubly periodic wave and folded solitary wave solutions for $(2+1)$-dimensional higher-order Broer-Kaup equation, Chaos Solitons Fractals, 31 (2007), 54-63.

[5] S. Kumar, K. Singh, R. K. Gupta, Painlev analysis, Lie symmetries and exact solutions for (2+1)-dimensional variable coefficients Broer-Kaup equations, Commun. Nonlinear Sci. Numer. Simul., 17 (2012), 1529-1541. 1

[6] D.-S. Li, Some new exact solutions to the $(2+1)$-dimensional Broer-Kaup equation with variable coefficients, J. At. Mol. Phys., 21 (2004), 133-138. 1

[7] B.-Q. Li, Y.-L. Ma, The non-traveling wave solutions and novel fractal soliton for the $(2+1)$-dimensional Broer-Kaup equations with variable coefficients, Commun. Nonlinear Sci. Numer. Simul., 16 (2011), 144-149.

[8] D.-S. Li, H.-Q. Zhang, Some new types of multisoliton solutions for the $(2+1)$-dimensional higher-order Broer-Kaup system, Appl. Math. Comput., 152 (2004), 847-853. 1

[9] S.-H. Ma, J.-P. Fang, Q.-B. Ren, Z. Yang, Chaotic behaviors of the $(2+1)$-dimensional generalized Breor-Kaup system, Chin. Phys. B, 21 (2012), 140-144.

[10] S.-H. Ma, Q.-B. Ren, J.-P. Fang, C.-L. Zheng, Special soliton structures and the phenomena of fission and annihilation of solitons for the $(2+1)$-dimensional Broer-Kaup system with variable coefficients, (Chinese) Acta Phys. Sinica, 56 (2007), 6777-6783. 1

[11] J.-Y. Qiang, S.-H. Ma, J.-P. Fang, Fusion and fission solitons for the $(2+1)$-dimensional generalized Breor-Kaup system, Chin. Phys. B, 19 (2010), 106-111. 1

[12] J.-H. Tian, New variable separation solutions to the $(2+1)$-dimensional Broer-Kaup equation, Science Technology and Engineering, 7 (2007), 5018-5021. 1

[13] H. Wang, Y.-H. Tian, Non-Lie symmetry groups and new exact solutions of a $(2+1)$-dimensional generalized Broer-Kaup system, Commun. Nonlinear Sci. Numer. Simul., 16 (2011), 3933-3940.

[14] Z. Yang, S.-H. Ma, J.-P. Fang, Chaotic solutions of $(2+1)$-dimensional Broek-Kaup equation with variable coefficients, Chin. Phys. B, 20 (2011), 33-37. 1

[15] E. Yomba, Y.-Z. Peng, Fission, fusion and annihilation in the interaction of localized structures for the (2+1)-dimensional generalized Broer-Kaup system, Chaos Solitons Fractals, 28 (2006), 650-657. 1

[16] J.-F. Zhang, P. Han, Generalized dromion structures of the $(2+1)$-dimensional Broer-Kaup equations, Chin. J. At. Mol. Phys., 18 (2001), 216-220. 1

[17] J.-F. Zhang, P. Han, Localized coherent structures of the $(2+1)$-dimensional Broer-Kaup equations, (Chinese) Acta Phys. Sinica, 51 (2002), 705-711.

[18] J.-F. Zhang, Y.-L. Liu, Localized coherent structures of the $(2+1)$-dimensional higher order Broer-Kaup equations, (Chinese) ; translated from Appl. Math. Mech., 23 (2002), 489-496, Appl. Math. Mech. (English Ed.), 23 (2002), 549-556.

[19] J.-L. Zhang, Y.-M. Wang, M.-L. Wang, Z.-D. Fang, Exact solutions to the $(2+1)$-dimensional Broer-Kaup equation with variable coefficients, Chin. J. At. Mol. Phys., 20 (2003), 92-94. 1

[20] S.-L. Zhang, B. Wu, S.-Y. Lou, Painlevé analysis and special solutions of generalized Broer-Kaup equations, Phys. Lett. A, 300 (2002), 40-48. 1

[21] X.-Q. Zhao, D.-B. Tang, L.-M. Wang, Y.-M. Zhang, Some new soliton wave solutions for (2 + 1)-dimensional Broer-Kaup equations, (Chinese) Acta Phys. Sin., 52 (2003), 1827-1831. 1 
[22] C.-L. Zheng, H.-P. Zhu, L.-Q. Chen, Exact solution and semifolded structures of generalized Broer-Kaup system in (2+1)-dimensions, Chaos Solitons Fractals, 26 (2005), 187-194. 1

[23] J.-M. Zhu, Z.-Y. Ma, New exact solutions to the $(2+1)$-dimensional Broer-Kaup equation, Chaos Solitons Fractals, 34 (2007), 476-481. 1

[24] J.-M. Zhu, Z.-Y. Ma, C.-L. Zheng, Localized fractal structure of the $(2+1)$-dimensional Broer-Kaup equations, (Chinese) Acta Phys. Sinica, 53 (2004), 3248-3251. 1 